Zwischen Lokaler Agenda und Global Village

\title{
Eine-Welt-Arbeit für Nachhaltigkeit
}

\author{
Nachhaltigkeit bedeutet auch die Verfolgung von Entwicklungszielen in interna- \\ tionaler Dimension. Im Rahmen der lokalen Agenden 21, einem der wichtigsten \\ Instrumente nationaler Entwicklung in Richtung Nachhaltigkeit, geht diese Di- \\ mension aber oft verloren. Dabei böten sich mannigfaltige Möglichkeiten lokale \\ und internationale Entwicklung zu koppeln.
}

Von Ellen Frings und Jürgen Wüst „Global denken - lokal handeln“, so eingängig dieser oft beanspruchte Leitspruch ist, so schwierig ist seine Umsetzung. Die aktuelle Nachhaltigkeitsdebatte ist, so die Experten im Diskursprozess des Rates für Nachhaltige Entwicklung, auf dem ,internationalen Auge“ blind und im lokalen Kontext verschärft sich diese Tendenz. Viele Lokale Agenda 21-Aktivitäten beschränken sich auf die Verbesserung der eigenen Lebensqualität (1). Dabei war gerade die globale Gerechtigkeit ein zentrales Anliegen, aus dem die Idee der nachhaltigen Entwicklung in den 80ern hervorging. Gut 20 Jahre später ist es dringender denn je: Einige Länder verlieren zunehmend den Anschluss an die Wirtschaftsentwicklung in der Welt, und die Kluft zwischen Arm und Reich wächst in vielen Länder.

Eine-Welt-Arbeit ist dabei kein neues Thema für Kommunen. In Agenda-Prozessen spielt sie aber nur dann eine Rolle, wenn sich die ,üblichen Verdächtigen" des Themas annehmen. Auf der Tagesordnung stehen meist Aktivitäten wie fair gehandelter Kaffee oder Informationsveranstaltungen. Dass eine breitere Verankerung selten gelingt, hat verschiedene Gründe: Globale Fragen liegen außerhalb unserer direkten Wahrnehmung und motivieren kaum zu freiwilligem Engagement - denn Engagement ist auch in der Lokalen Agenda dort am größten, wo persönliche Interessen berührt sind. Außerdem beeinträchtigt das Negativsyndrom die Attraktivität des Themas (2). Die Medien berichten meist im Katastrophenfall über Entwicklungsländer und liefern vorwiegend Bilder der Chancenlosigkeit.

Dabei hat Eine-Welt-Arbeit viel mehr direkte Bezüge zu unserem Alltag als es zunächst scheint. Über Produkte, Wirtschaftsbeziehungen, Medien und Tourismus sind entfernte Lebensräume im
„Global Village“ zusammengerückt, und in einer durch Zuwanderung geprägten Gesellschaft begegnen sich verschiedene Kulturen täglich. EineWelt-Arbeit wird erst dann aus ihrer Nische herausfinden, wenn sie diese Alltagsbezüge herstellt und den Dialog zwischen den Kulturen als Voraussetzung für eine lernende Gesellschaft und für eine humane und zukunftsfähige Gestaltung der Globalisierung versteht (3). Hier kann die Lokale Agenda 21 ansetzen, indem sie Verständigung fördert und den Zuwachs an Kompetenzen und Chancen in den Mittelpunkt stellt.

\section{Beispiel Kinder und Jugendliche}

Kaum ausgeschöpft wurden bisher die Potentiale, die das Bildungssystem für die Umsetzung von Nachhaltigkeit bietet. Dabei gibt es genug praktische Erfahrungen, beispielsweise aus dem Bund-Länder-Programm. Viele Reformen, die seit der Veröffentlichung der PISA-Studien gefordert werden, sind bereits in den Konzepten zur Bildung für Nachhaltigkeit antizipiert (4). In der aktuellen Diskussion um Bildungsreformen spielt Nachhaltigkeit jedoch kaum eine Rolle, so auch nicht bei den laufenden Reformen im Bereich der frühkindlichen Bildung. Dabei wäre der Kindergarten der beste Ansatzpunkt. Kinder aus verschiedenen Kulturen treffen hier erstmals aufeinander, und von allen Bildungseinrichtungen hat er die größte Nähe zum Elternhaus. Zahlreiche Aktivitäten sind möglich. Migranten können über ihre Kultur berichten und beim Aufbau von Partnerschaften Kontakte herstellen. Die Feste im Jahresverlauf bieten auch Anlässe, die Traditionen und Rituale der verschiedenen Kulturen einzubeziehen. Die natürliche Neugier der Kinder führt zu täglich neuen Fragen, denen gemeinsam nachgegangen werden kann: Was steckt alles in der Schokolade? Wer produziert unser Spielzeug? Oft bleiben die Fragen aber unbeantwortet, weil den pädagogischen Fachkräften das Wissen fehlt. Gemeinsam mit Eltern, Unternehmen und lokalen Initiativen können solche Kompetenzen in die pädagogische Arbeit eingebunden werden.

Die große Herausforderung besteht zukünftig in der Integration von Bildung und Nachhaltigkeit. Statt Einzelaktivitäten gilt es unterschiedliche Kompetenzen in vernetzten Prozessen zu fördern. Wenn Kinder per Internet Kontakt zu Kindern in einem anderen Land aufnehmen, werden mediale, soziale und interkulturelle Kompetenzen gefördert. Die Lokale Agenda wäre ein geeigneter Rahmen, um die von den Vereinten Nationen ausgerufene Dekade der „Bildung für Nachhaltigkeit“ (2005-2014) aktiv auszugestalten. Der Einstieg könnte ein pädagogischer Tag zum Thema Nachhaltigkeit sein, der die Bildungseinrichtungen vor Ort zusammenführt und die Basis für konkrete Projekte liefert.

\section{Beispiel Vereine und Unternehmen}

Eine-Welt-Themen haben für viele Vereine auf den ersten Blick wenig mit ihrer Arbeit zu tun. Dabei liegen die Bezugspunkte manchmal direkt vor den Füßen. Dass viele Fußbälle das Produkt von Kinderarbeit waren, wurde bis zur Fair Play - Fair Pay-Kampagne der Clean Clothes Campaign kaum wahrgenommen. Auch der Einstieg in den „privaten Emissionshandel“, etwa bei Veranstaltungen und Festen, könnte das Bewusstsein für Nachhaltigkeitsthemen im Verein schärfen. Dabei werden die Treibhausemissionen durch Verkehr, Catering oder Heizung mit einer Investition in ein Energiespar-Projekt in einem Entwicklungsland ausgeglichen. So könnten Sponsoren finanzielle Zuschüsse mit der Bedingung einer solchen Kompensationszahlung verbinden. Interessante Möglichkeiten bietet auch der Austausch in Städtepartnerschaften mit Entwicklungsländern oder die Patenschaft bei Projekten lokaler Eine-Welt-Initiativen. Vereine haben außerdem eine hohe Bedeutung für den interkulturellen Austausch am Ort. Die gezielte Einbindung von Migranten oder Kooperationen mit Vereinen von Bürgern ausländischer Herkunft sind weitere Ansatzpunkte. In den letzten Jahren haben sich vor allem Großunternehmen mit Corporate Social Responsibility und nachhaltiger Unternehmensfuihrung befasst. Kleine und mittlere Unternehmen (KMU), so die häufige Meinung, können kaum auf globale $\mathrm{Zu}$ - 
sammenhänge einwirken. Dass Wirtschaftskooperationen auf lokaler Ebene aber möglich sind, zeigte das Projekt ,Nachhaltiges Wirtschaften Heidelberg“. Hier wurde die Kooperation zwischen einer lokalen Initiative mit langjährigem Engagement in Ruanda und Heidelberger Betrieben angestoßen. Die Motivation der Unternehmen war erstaunlich hoch, die Partner in Ruanda beim Aufbau einer effizienten Holzwirtschaft, etwa durch Materialien, Werkzeuge oder Know-how zu unterstützen. Sicher nicht zuletzt aufgrund der thematischen Nähe und des regionalen Bezuges der Initiative (5). Weitere Handlungsmöglichkeiten in den Betrieben sind die Beschäftigung von ausländischen Stipendiaten und Praktikanten oder die Förderung interkulturellen Lernens.

\section{Beispiel Politik und Verwaltung}

Vielfältige Möglichkeiten, Eine-Welt-Themen stärker in der Gesellschaft zu verankern, bieten Städtepartnerschaften. Der Austausch mit Entwicklungsländern ermöglicht Erleben jenseits moralischer Ansprüche und erreicht andere Zielgruppen als die klassische Eine-Welt-Arbeit. Gleichzeitig sind Städtepartnerschaften ein Rahmen, um die Kompetenzen der kommunalen Verwaltungen weiterzugeben, etwa durch Beratung bei Verwaltungsfragen oder durch Unterstïtzung bei partizipativer Problembearbeitung. Die Anwendungsbereiche vor Ort sind vielfältig: Aufbau von Verwaltungen oder sozialen Einrichtungen wie Gemeindebüchereien, Lösung von Wasserkonflikten durch Dialog und Mediation, Finanzierung regenerativer Energiegewinnung durch Public Private Partnership (PPP). Dabei spielt die Vermittlung von Informationen zur Selbsthilfe eine wichtige Rolle. Mögliche Fragen betreffen Finanzierungsmöglichkeiten in der Entwicklungszusammenarbeit oder Best-Practices bei Bürgerbeteiligung. InWEnt fördert den Nord-Süd-Wissensaustausch jährlich mit dem „Exposure“-Programm. Fachund Führungskräfte aus Verwaltung und Unternehmen in Entwicklungsländern erhalten hier die Möglichkeit, sich mit ihren deutschen ,counterparts" über regionale Wirtschaftsförderung auszutauschen. Dass sich Entwicklungszusammenarbeit in Kommunen auch institutionell stärker verankern lässt, zeigt die Stadt Bonn mit einem eigenen Ausschuss für internationale Beziehungen und Lokale Agenda, der unter anderem Projekte in Usbekistan, China, Bolivien und der Mongolei begleitet.

\section{Ausblick}

Durch lokale Bezüge wird Eine-Welt-Arbeit fassbar, und damit birgt die Lokale Agenda 21 erhebliche Potentiale, den Wandel zu einer nachhaltigen Entwicklung von unten zu befördern. Gute Beispiele sowie ausbaubare Ansätze sind vorhanden. Dazu bedarf es aber auf mindestens zwei Ebenen eines Perspektivenwechsels: vom Engagement aus Schuldgefühlen hin zu einem partnerschaftlichen Ansatz und von einer Lokalen Agenda 21 als partizipativem Breitenprozess hin zu einer Lokalen Agenda der strategischen Allianzen. Notwendig sind dazu Promotoren in der Verwaltung, in Agenda- oder Eine-Welt-Initiativen, die die Bedeutung des Themas für eine globale nachhaltige Entwicklung erkennen. Ihre Aufgabe ist es, neue, geeignete Kooperationspartner in Verwaltung oder Politik ebenso wie in Vereinen, Schulen, Verbänden der Wirtschaft oder in Unternehmen zu suchen. Eine systematische Auswertung der lokalen Akteurslandschaft mit Blick auf thematische Ansatzpunkte und vorhandene Potenziale über die bisherigen Rollenverteilungen hinaus kann dabei den Anfang machen.

\section{Anmerkungen}

(1) Frings, E. et al.: Lokale Agenda 21 im Kontext der kommunalen Steuerungsinstrumente. UBA-Texte 34/02, Berlin 2002

(2) Wilmsen, Ch.: Agenda 21 - Lokale Agenda - Nachhaltigkeit - Was ist denn das? In: Lokale Agenda und öffentliche Verwaltung. Nachhaltigkeit und Globalisierung als Element der Verwaltungsreform. 2002, S. 6-9.

(3) Nitschke, U.: Chancen kommunaler Entwicklungszusammenarbeit. Lernen im Nord-Süd-Dialog. In: Aus Politik und Zeitgeschichte. H. 15-16, 2004.

(4) de Haan, G.: Schulprogramm BLK 21 - die Antwort auf PISA. In: punktum 2/2002, S. 15.

(5) Frings, E.: Nachhaltiges Wirtschaften Heidelberg. In: Alternative Kommunalpolitik. Heft 2, 2003, S. $42 \mathrm{ff}$.

\section{Die Autorlnnen}

Ellen Frings und Dr. Jürgen Wüst sind Projektleiter am Institut für Organisationskommunikation (IFOK). Kontakt: IFOK, Berliner Ring 89, 64625 Bensheim. Tel. 06251-84160, E-Mail: ellen.frings@ifok.de, juergen.wuest@ifok.de

\section{ökom verlag - GAIA 2/04}

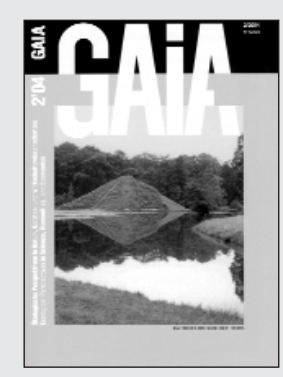

Zur Zukunftsfähigkeit der Umweltpolitik

Ist die Umweltpolitik auf dem Weg in die bürokratisch und politisch gefestigte Belanglosigkeit? Oder gelingt es ihr, sich den gesellschaftlichen Herausforderungen zu stellen? Die aktuelle GAIA-Ausgabe zeigt unter anderem, wie sich die Umweltpolitik hin zu einer Zukunftspolitik verändern kann.

Lesen Sie außerdem:

_Abschied von der einen Natur (W. Zierhofer)

Die formative Szenario-Analyse in der partizipativen Regionalentwicklung (R. Kirchner-Heßler)

_Integration von ökologischem Wissen in die Raumplanung (P. Müller, S. Güsewell und K.M. Tanner)

GAIA - Ökologische Perspektiven in Natur-, Geistes- und Wirtschaftswissenschaften

_veröffentlicht begutachtete Originalarbeiten aus der Umweltforschung

GAIA ist jetzt im Volltext verfügbar unter www.ingentaselect.com

Jahresabonnement von 4 Ausgaben:

Einzelpersonen: $79,90 € /$ Institutionen: $144,90 € /$ Studenten: $51,50 €$

Einzelheft: 20,- $€$ (zzgl. Versandkosten)

Erhältlich bei

CONSODATA ONE-TO-ONE, Semmelweisstraße 8, D-82152 Planegg

Fon $++49 /(0) 89 / 857$ 09-155, Fax -131, kontakt@oekom.de

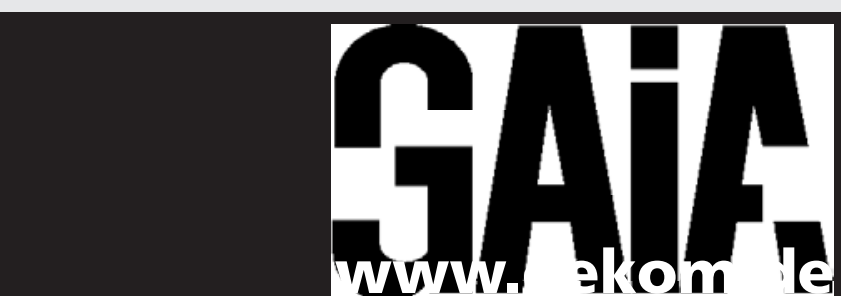


(c) 20I0 Authors; licensee IÖW and oekom verlag. This is an article distributed under the terms of the Creative Commons Attribution Non-Commercial No Derivates License (http://creativecommons.org/licenses/by-nc-nd/3.o/), which permits unrestricted use, distribution, and reproduction in any medium, provided the original work is properly cited. 